\title{
Dieta del Pirincho (Guira guira) en el nordeste de la provincia de Buenos Aires, Argentina (Cuculiformes: Cuculidae)
}

\author{
Guillermo E. Soave ${ }^{1}$, Carlos A. Darrieu ${ }^{1}$, María E. Aribalzaga ${ }^{1}$, Aníbal R. Camperi $^{1}$, \\ Mariano Lucía $^{2}$, Jorge Williams ${ }^{3} \&$ Marcos Juarez $^{1}$ \\ 1. Sección Ornitología, División Zoología Vertebrados y Cátedra de Ornitología, Facultad de Ciencias Naturales y \\ Museo de La Plata, Paseo del Bosque, 1900 La Plata, Buenos Aires, Argentina; gsoave@fcnym.unlp.edu.ar \\ 2. División Entomología, Facultad de Ciencias Naturales y Museo, Universidad Nacional de La Plata, Paseo del Bosque, \\ 1900 La Plata, Buenos Aires, Argentina; mlucia@fcnym.unlp.edu.ar \\ 3. Cátedra de Herpetología, Facultad de Ciencias Naturales y Museo, Universidad Nacional de La Plata, Paseo del \\ Bosque, 1900 La Plata, Buenos Aires, Argentina; williams@fcnym.unlp.edu.ar
}

Recibido 14-II-2008. Corregido 30-VI-2008. Aceptado 31-VII-2008.

\begin{abstract}
Feeding ecology of the Guira Cuckoo (Guira guira) in the northeastern Buenos Aires Province. We studied the diet and feeding ecology of the Guira Cuckoo (Guira guira), a bird that has a wide distribution in Argentina. We analyzed 62 stomachs, obtained between 1991 and 1997 (26 in spring, 13 in summer, 10 in autumn and 13 in winter), in the localities La Balandra and Punta Blanca, Magdalena district, Buenos Aires province. In order to establish the contribution of each prey item, we applied the relative importance index. The dietary contribution in terms of number and volume was also taken into account. This species feeds only on animals. The trophic spectrum was determined on the basis of the identification of 92 items, all of animal origin, principally Insecta (98 \%), Annelida, Mollusca, Arachnida, Crustacea, Amphibia, Reptilia and Aves. In conclusion, G. guira is a opportunistic species that consumes animal preys exclusively. Rev. Biol. Trop. 56 (4): 1883-1892. Epub 2008 December 12.
\end{abstract}

Key words: Guira Cuckoo, Guira guira, diet, Buenos Aires, Argentina.

El Pirincho (Guira guira) es un ave residente, cuya distribución abarca gran parte de América del Sur, desde el este de Brasil (desde el Amazonas hasta Mato Grosso y Río Grande do Sul), sudeste de Bolivia, todo el Paraguay y el Uruguay, y desde el norte hasta el centro de la Argentina (Del Hoyo et al. 1997).

En nuestro país se distribuye desde Salta y Jujuy hasta la provincia del Chubut (donde se ha expandido a partir de la década de 1950), abarcando las regiones mesopotámica y chacopampeana, y el centro y oeste del país hasta los 2000 metros de altura en la zona cordillerana. Es probable que las modificaciones introducidas por el hombre en la zona pampeana hayan ampliado su área de dispersión, ya que la implantación de árboles (inexistentes en el antiguo ecosistema de la región) podría haber permitido su avance luego de la colonización agrícola. Su hábitat incluye tanto campos y llanuras como las sierras del centro del país y las elevaciones andinas, donde prefiere frecuentar sabanas abiertas con matorrales y árboles para refugiarse durante la noche y nidificar. Además se lo encuentra en áreas urbanas y suburbanas.

De hábitos gregarios, conviven habitualmente entre diez y veinte individuos. Generalmente se alimentan en bandada, caminando en busca de sus presas, mientras que un miembro del grupo permanece vigilando desde la rama de un árbol, para avisar sobre la presencia de algún depredador. Incluso suelen agruparse durante la época de nidificación, y varias hembras pueden depositar sus huevos en un mismo nido. 
La marcada voracidad de los pirinchos cumple una importante función reguladora. Marcadamente animalívoros, en sus contenidos estomacales las semillas sólo se registran en forma casual. Su alimento principal lo constituyen los insectos y sus larvas, para atrapar a los cuales a veces se desplaza en grupo detrás de arados y rastras. De esta forma, se ha creado una asociación útil en la que tanto el hombre como el Pirincho resultan beneficiados. El ave regula la población de insectos perjudiciales para el cultivo, y a la vez explota la capacidad mecánica del ser humano que, en la labor agrícola, le ofrece abundancia de insectos y, en su avance colonizador, foresta áreas de pastizal ampliando el área de distribución de esta especie.

Este trabajo tiene como objetivos conocer el espectro trófico de Guira guira a lo largo de un ciclo anual, registrar sus posibles variaciones estacionales y determinar la fidelidad ambiental en relación con su alimentación.

\section{ÁREA DE ESTUDIO Y MÉTODOS}

Las muestras fueron obtenidas durante 20 salidas al campo realizadas entre 1991 y 1997, en una zona caracterizada por la presencia de bosques en galería, talares, áreas abiertas rurales y semirurales, entre las localidades de La Balandra y Punta Blanca, Partido de Magdalena, Provincia de Buenos Aires, Argentina ( $34^{\circ} 56^{\prime} 22^{\prime \prime} \mathrm{S}-57^{\circ} 43^{\prime} 11^{\prime \prime} \mathrm{W}$ ). En el presente trabajo se estudiaron ejemplares capturados con armas de fuego, que primariamente fueron utilizados para estudios endoectoparásitológicos y de contaminantes. Fueron revisados 62 estómagos (26 de primavera, 13 de verano, 10 de otoño y 13 de invierno). De los especímenes capturados se registraron datos de peso corporal, sexo y estado gonadal. Los estómagos fueron extraídos en el campo y fijados en alcohol $70 \%$ inmediatamente después de la captura. En el laboratorio se separaron bajo lupa binocular, los restos de los distintos organismos. Este material fue determinado al menor nivel taxonómico posible, cuantificado y pesado en balanza digital.
Para estimar el Ancho del Nicho Trófico (FNB) se utilizó el índice de Levins (1968). $\mathrm{FNB}=1 /\left(€ \mathrm{pi}^{2}\right)$, donde $p i$ es la proporción de la presa $i$ en la dieta. Un valor estandarizado (FNBst) varió entre 0 y 1 y fue calculado de acuerdo a la siguiente fórmula: $\mathrm{FNBst}=(\mathrm{FNB}-$ 1) / (n-1), donde $n$ es el número total de categorías presa (Colwell y Futuyma 1971).

\section{RESULTADOS}

Todos los estómagos analizados $(\mathrm{N}=62)$ contenían alimento. El peso medio de las muestras fue $4.9 \mathrm{~g}(\mathrm{DS} \pm 3.96 ; \mathrm{r}=0.7-16.1 ; \mathrm{N}=62)$ con un promedio de 27 ítems presa ( $\mathrm{DS} \pm 32$; $\mathrm{N}=1.661$ ). Donde $\mathrm{N}$ es número de estómagos, DS desvío estándar y $\mathrm{r}$ ámbito. Dado que entre machos y hembras no se encontraron diferencias significativas en el número y/o el peso de las presas consumidas ( $U$-Mann Whitney, n.s), ambos sexos fueron considerados en conjunto.

El espectro trófico basado en la identificación de 93 taxones correspondientes en su totalidad a la fracción animal (ver Apéndice), estuvo compuesto por: Insecta (69), Arachnida (8), Crustacea (1), Mollusca (3), Anellida (1), Amphibia (7), Reptilia (3) y Aves (1).

El Cuadro 1 muestra la frecuencia de presencia, importancia numérica y peso para los distintos ítems presa agrupados en taxones mayores.

Los insectos, hallados en 61 muestras $(\mathrm{N}$ $=62$ ), constituyeron las principales presas a través de todo el período de muestreo, contribuyendo en casi el $90 \%$ en número y poco

CUADRO 1

Frecuencia de presencia, importancia numérica y peso de los items presa agrupados en taxa mayores

$\begin{array}{lccc} & \text { Número } & \text { Frecuencia } & \text { Peso } \\ \text { Insectos } & 1444 & 61 & 178 \\ \text { Arácnidos } & 104 & 39 & 17,9 \\ \text { Otros Invertebrados } & 17 & 8 & 10,2 \\ \text { Anfibios } & 39 & 21 & 51,5 \\ \text { Reptiles } & 10 & 10 & 25,8 \\ \text { Aves } & 5 & 4 & 11,1\end{array}$


menos del $60 \%$ en peso, de la masa total consumida. Orthoptera y Lepidoptera resultaron ser las principales presas de este grupo. Ambos órdenes en conjunto constituyeron casi el 60 $\%$ en número y cerca del $90 \%$ en peso. Los Lepidoptera estuvieron representados principalmente por estadios preimaginales (larvas y pupas), mientras que las langostas constituyeron las principales presas de Orthoptera. Le siguen en orden de importancia Hymenoptera y Coleoptera, aunque su contribución en peso es mucho menor (Fig. 1 y Cuadro 2).

Otros invertebrados estuvieron representados por Arachnida, Crustacea, Mollusca y Anellida, constituyendo ítems de menor cuantía tanto en número como en peso.

Por su parte los vertebrados estuvieron presentes en bajo número (54 individuos), pero constituyeron un $30 \%$ del peso total de la muestra.

Los Amphibia presentaron la mayor importancia relativa tanto en número $(72.2 \%)$, peso $(58.3 \%)$ y cantidad de taxones (Cuadro 1 y Cuadro 2). En general se trató de individuos de pequeño tamaño, en su mayoría juveniles, no registrándose estadios larvales.
Ophiodes vertebralis fue la presa más común entre los Reptilia, mientras que las Aves estuvieron representadas por un huevo, un pichón y restos óseos que no pudieron ser determinados.

El número de presas consumidas por estación no varió significativamente (KruskalWallis, n.s.), aunque se encontraron diferencias en el peso (Kruskal-Wallis, $p=0.0190$ ); las muestras más pesadas en promedio se registraron en otoño y principalmente invierno (Fig. 2).

La amplitud del nicho trófico fue más alta en primavera y verano $(\mathrm{FNBst}=0.14$ y 0.10$)$ que en otoño e invierno (FNBst $=0.07$ y 0.02 ), coincidiendo esto con la disminución de especies capturadas (desde 69 en primavera hasta 29 en invierno).

\section{DISCUSIÓN}

Existen pocas contribuciones sobre la dieta de esta especie, las cuales aportan solamente datos aislados, anecdóticos $\mathrm{y} / \mathrm{o}$ con muestreos insuficientes. Marelli (1919) analiza el contenido estomacal de ejemplares obtenidos

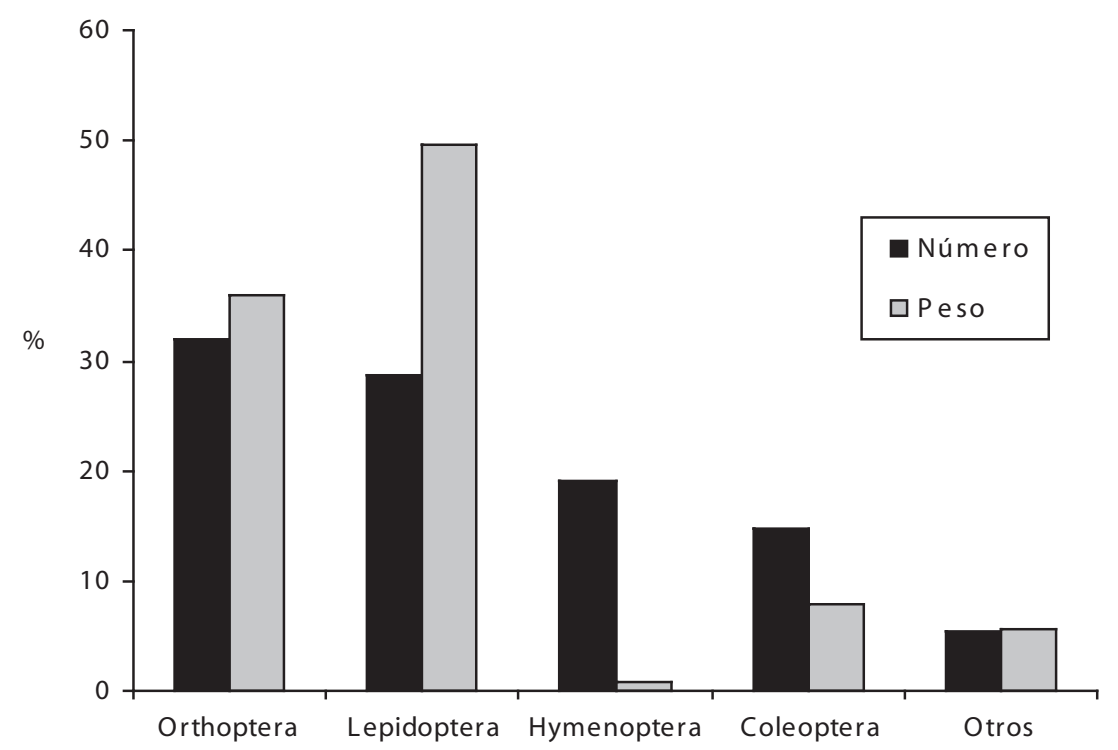

Fig. 1. Contribución de los órdenes de Insecta en términos de número y peso. 
CUADRO 2

Lista de los items que integran la dieta de Guira guira durante un ciclo anual

\begin{tabular}{cccccccccc} 
& \multicolumn{2}{c}{ PRIMAVERA } & \multicolumn{2}{c}{ VERANO } & & \multicolumn{2}{c}{ OTONO } & \multicolumn{2}{c}{ INVIERNO } \\
ÍTEMS PRESA & $\mathrm{N}$ & $\mathrm{F}$ & $\mathrm{N}$ & $\mathrm{F}$ & $\mathrm{N}$ & $\mathrm{F}$ & $\mathrm{N}$ & $\mathrm{F}$
\end{tabular}

INSECTA

COLEOPTERA

Carabidae

Scarites melanarius

14.3

Scarites anthracinus

14.3

$\begin{array}{ll}2 & 7.7\end{array}$

Trirammatus striatulus

$4 \quad 8.7$

Aspidoglossa intermedia

14.3

Apenes aenea

14.3

Scarites sp.

24.3

Colossoma sp.

$\begin{array}{ll}7 & 13.0\end{array}$

Carabidae sp.

$\begin{array}{ll}3 & 8.7\end{array}$

2

12.5

Curculionidae

Calendra sp.

Driophthorinae sp.

Calendrinae sp.

Curculionidae sp.

Scarabeidae

Dyscinetus sp.

Lygirus sp.

Stenocrates sp.

Dynastinae sp.

Scarabeidae sp.

Tenebrionidae

Tenebrionidae sp.

Aphodiidae

Aphodiidae sp.

Coccinellidae

Cycloneda sanguinea

$1 \quad 4.3$

Elateridae

Elateridae sp.

4

8.7

Dytiscidae

Dytiscidae sp.

$10 \quad 17.4$

6.2

$1 \quad 6$

$\begin{array}{llll}8 & 17.4 & 4 & 18.7\end{array}$

$5 \quad 18.7$

$3 \quad 10.0$

$2 \quad 7.7$

14.3

$\begin{array}{lllll}18.7 & 8 & 30.0 & 5 & 15.4\end{array}$

$\begin{array}{llllll}16 & 30.4 & 10 & 18.7 & 5 & 20.0\end{array}$

Chrysomelidae

Chrysomelidae sp.

4

12.5

Hydrophilidae

Hydrophilidae sp.

3

6.2

Dermestidae

Dermestes sp.

$1 \quad 10.0$ 
CUADRO 2 (Continued)

Lista de los items que integran la dieta de Guira guira durante un ciclo anual

\begin{tabular}{|c|c|c|c|c|c|c|c|c|}
\hline \multirow{2}{*}{ ÍTEMS PRESA } & \multicolumn{2}{|c|}{ PRIMAVERA } & \multicolumn{2}{|c|}{ VERANO } & \multicolumn{2}{|c|}{ OTOÑO } & \multicolumn{2}{|c|}{ INVIERNO } \\
\hline & $\mathrm{N}$ & $\mathrm{F}$ & $\mathrm{N}$ & $\mathrm{F}$ & $\mathrm{N}$ & $\mathrm{F}$ & $\mathrm{N}$ & $\mathrm{F}$ \\
\hline Coleoptera sp. & 23 & 21.7 & 18 & 25.0 & 7 & 10.0 & 21 & 61.5 \\
\hline \multicolumn{9}{|l|}{ HEMIPTERA } \\
\hline \multicolumn{9}{|l|}{ Pentatomidae } \\
\hline Nezara viridula & & & & & 1 & 10.0 & & \\
\hline Edessa $\mathrm{sp}$ & 8 & 13.0 & 2 & 12.5 & 1 & 10.0 & 2 & 7.7 \\
\hline Pentatomidae sp. & 3 & 4.3 & 2 & 6.2 & 1 & 10.0 & & \\
\hline \multicolumn{9}{|l|}{ Coreidae } \\
\hline Corecoris sp. & 1 & 4.3 & & & & & & \\
\hline Coreidae sp. & 2 & 4.3 & & & & & 2 & 7.7 \\
\hline \multicolumn{9}{|l|}{ Belostomatidae } \\
\hline Belostoma sp. & 1 & 4.3 & & & 2 & 20.0 & & \\
\hline \multicolumn{9}{|l|}{ Cicadidae } \\
\hline Cicadidae sp. & 6 & 4.3 & & & & & 3 & 7.7 \\
\hline \multicolumn{9}{|l|}{ Membracidae } \\
\hline Membracidae sp. & & & & & & & 2 & 7.7 \\
\hline Hemiptera sp. & 2 & 8.7 & 1 & 6.2 & & & 2 & 7.7 \\
\hline \multicolumn{9}{|l|}{ ORTHOPTERA } \\
\hline \multicolumn{9}{|l|}{ Acrididae } \\
\hline Dichroplus sp. & 5 & 8.7 & 71 & 25.0 & 11 & 30.0 & & \\
\hline Allotruxalis sp. & 29 & 4.3 & 29 & 12.5 & & & & \\
\hline Gomphocorinae sp. & 36 & 4.3 & & & & & & \\
\hline Acridinae sp. & 40 & 4.3 & & & & & & \\
\hline Acrididae sp. & 4 & 39.1 & 52 & 37.5 & 12 & 20.0 & & \\
\hline \multicolumn{9}{|l|}{ Gryllidae } \\
\hline Gryllidae sp. & 2 & 8.7 & 1 & 6.2 & 3 & 20.0 & & \\
\hline \multicolumn{9}{|l|}{ Proscopidae } \\
\hline Proscopidae sp. & 1 & 4.3 & 3 & 6.2 & 2 & 20.0 & & \\
\hline \multicolumn{9}{|l|}{ Tettigonidae } \\
\hline Tettigonidae sp. & & & 2 & 6.2 & 1 & 10.0 & & \\
\hline \multicolumn{9}{|l|}{ Grillotalpidae } \\
\hline Grillotalpidae sp. & & & 2 & 6.2 & & & & \\
\hline Orthoptera sp. & 26 & 26.0 & 44 & 37.5 & 40 & 60.0 & 9 & 23.0 \\
\hline \multicolumn{9}{|l|}{ LEPIDOPTERA } \\
\hline \multicolumn{9}{|l|}{ Saturnidae } \\
\hline Hylesia nigricans & 78 & 4.3 & & & & & & \\
\hline Hemileucinae sp. & 4 & 13.0 & 6 & 18.7 & 26 & 40.0 & 13 & 15.4 \\
\hline \multicolumn{9}{|l|}{ Noctuidae } \\
\hline Noctuidae sp. & 1 & 4.3 & & & & & & \\
\hline
\end{tabular}


CUADRO 2 (Continued)

Lista de los items que integran la dieta de Guira guira durante un ciclo anual

\begin{tabular}{|c|c|c|c|c|c|c|c|c|}
\hline \multirow{2}{*}{ ÍTEMS PRESA } & \multicolumn{2}{|c|}{ PRIMAVERA } & \multicolumn{2}{|c|}{ VERANO } & \multicolumn{2}{|c|}{ OTOÑO } & \multicolumn{2}{|c|}{ INVIERNO } \\
\hline & $\mathrm{N}$ & $\mathrm{F}$ & $\mathrm{N}$ & $\mathrm{F}$ & $\mathrm{N}$ & $\mathrm{F}$ & $\mathrm{N}$ & $\mathrm{F}$ \\
\hline \multicolumn{9}{|l|}{ Sphingidae } \\
\hline Sphingidae sp. & 12 & 26.0 & & & 3 & 10.0 & & \\
\hline \multicolumn{9}{|l|}{ Arctiidae } \\
\hline Arctiidae sp. & & & 2 & 6.2 & & & 7 & 7.7 \\
\hline Lepidoptera sp. & 16 & 26.0 & 3 & 12.5 & 1 & 10.0 & 237 & 69.2 \\
\hline \multicolumn{9}{|l|}{ ODONATA } \\
\hline \multicolumn{9}{|l|}{ Aeshnidae } \\
\hline Aeshna sp. & 1 & 4.3 & & & & & & \\
\hline Anisoptera sp. & 1 & 4.3 & 2 & 12.5 & & & & \\
\hline Odonata sp. & 2 & 4.3 & & & & & & \\
\hline \multicolumn{9}{|l|}{ HYMENOPTERA } \\
\hline \multicolumn{9}{|l|}{ Vespidae } \\
\hline Vespidae sp. & 1 & 4.3 & & & & & & \\
\hline \multicolumn{9}{|l|}{ Apidae } \\
\hline Bombus atratus & & & 1 & 6.2 & & & & \\
\hline \multicolumn{9}{|l|}{ Formicidae } \\
\hline Solenopsis sp. & 111 & 8.7 & 75 & 12.5 & 65 & 20.0 & & \\
\hline Acromyrmex sp. & 4 & 4.3 & 10 & 18.7 & & & & \\
\hline Mycocepurus sp. & 2 & 4.3 & & & & & & \\
\hline Atta sp. & 1 & 4.3 & & & & & & \\
\hline Formicidae sp. & & & 20 & 12.5 & & & 1 & 7.7 \\
\hline \multicolumn{9}{|l|}{ TRICHOPTERA } \\
\hline Trichoptera sp. & 1 & 4.3 & 1 & 6.2 & & & 1 & 7.7 \\
\hline \multicolumn{9}{|l|}{ DIPTERA } \\
\hline \multicolumn{9}{|l|}{ Tabanidae } \\
\hline Tabanidae sp. & & & 1 & 6.2 & & & & \\
\hline \multicolumn{9}{|l|}{ Tachinidae } \\
\hline Tachinidae sp. & & & & & 1 & 10.0 & & \\
\hline \multicolumn{9}{|l|}{ Stratyomyidae } \\
\hline Stratyomyidae sp. & & & 1 & 6.2 & & & & \\
\hline \multicolumn{9}{|l|}{ Muscidae } \\
\hline Stomoxys calcitrans & & & & & 1 & 10.0 & & \\
\hline Diptera sp. & 2 & 8.7 & & & & & 11 & 7.7 \\
\hline \multicolumn{9}{|l|}{ MANTODEA } \\
\hline \multicolumn{9}{|l|}{ Mantidae } \\
\hline Mantidae sp. & 1 & 4.3 & 4 & 25.0 & 1 & 10.0 & & \\
\hline \multicolumn{9}{|l|}{ BLATTODEA } \\
\hline Blattodea sp. & & & 1 & 6.2 & & & 1 & 7.7 \\
\hline
\end{tabular}


CUADRO 2 (Continued)

Lista de los items que integran la dieta de Guira guira durante un ciclo anual

\begin{tabular}{|c|c|c|c|c|c|c|c|c|}
\hline \multirow{2}{*}{ ÍTEMS PRESA } & \multicolumn{2}{|c|}{ PRIMAVERA } & \multicolumn{2}{|c|}{ VERANO } & \multicolumn{2}{|c|}{ OTOÑO } & \multicolumn{2}{|c|}{ INVIERNO } \\
\hline & $\mathrm{N}$ & $\mathrm{F}$ & $\mathrm{N}$ & $\mathrm{F}$ & $\mathrm{N}$ & $\mathrm{F}$ & $\mathrm{N}$ & $\mathrm{F}$ \\
\hline \multicolumn{9}{|l|}{ ARACHNIDA } \\
\hline \multicolumn{9}{|l|}{ ARANEIDA } \\
\hline \multicolumn{9}{|l|}{ Lycosidae } \\
\hline Lycosidae sp. & 24 & 65.2 & 19 & 56.2 & 4 & 20.0 & 20 & 69.2 \\
\hline \multicolumn{9}{|l|}{ Tetragnathidae } \\
\hline Tetragnathidae sp. & 1 & 4.3 & & & & & & \\
\hline \multicolumn{9}{|l|}{ Salticidae } \\
\hline Salticidae sp. & 2 & 8.7 & & & & & & \\
\hline Araneida sp. & 1 & 4.3 & 2 & 12.5 & 14 & 40.0 & & \\
\hline \multicolumn{9}{|l|}{ OPILIONIDA } \\
\hline \multicolumn{9}{|l|}{ Gonyleptidae } \\
\hline Acanthopachylus aculeatus & 3 & 8.7 & 1 & 6.2 & & & & \\
\hline Opilionida sp. & 14 & 26.0 & 3 & 6.2 & 2 & 20.0 & 22 & 23.0 \\
\hline \multicolumn{9}{|l|}{ ESCORPIONIDA } \\
\hline Escorpionidae sp. & 1 & 4.3 & & & & & & \\
\hline \multicolumn{9}{|l|}{ ACARINA } \\
\hline Acarina sp. & 13 & 4.3 & & & & & & \\
\hline \multicolumn{9}{|l|}{ CRUSTACEA } \\
\hline \multicolumn{9}{|l|}{ ISOPODA } \\
\hline Isopoda sp. & 1 & 4.3 & & & 2 & 10.0 & & \\
\hline \multicolumn{9}{|l|}{ GASTROPODA } \\
\hline \multicolumn{9}{|l|}{ SYSTELLOMMATOPHORA } \\
\hline \multicolumn{9}{|l|}{ Veronicellidae } \\
\hline Veronicellidae sp. & 2 & 8.7 & & & & & 6 & 15.4 \\
\hline \multicolumn{9}{|l|}{ BASOMMATOPHORA } \\
\hline \multicolumn{9}{|l|}{ Planorbidae } \\
\hline Planorbidae sp. & & & 1 & 6.2 & & & & \\
\hline Gastropoda sp. & 2 & 4.3 & & & & & & \\
\hline \multicolumn{9}{|l|}{ CLITELLATA } \\
\hline \multicolumn{9}{|l|}{ LUMBRICULIDA } \\
\hline \multicolumn{9}{|l|}{ Lumbricidae } \\
\hline Lumbricidae sp. & & & & & & & 3 & 7.7 \\
\hline \multicolumn{9}{|l|}{ AMPHIBIA } \\
\hline ANURA & & & & & & & & \\
\hline Leptodactylidae & & & & & & & & \\
\hline Leptodactylus latinasus & 1 & 4.3 & & & 1 & 10.0 & 1 & 7.7 \\
\hline Leptodactylus sp. & 4 & 17.4 & 1 & 6.2 & 5 & 20.0 & & \\
\hline
\end{tabular}


CUADRO 2 (Continued)

Lista de los items que integran la dieta de Guira guira durante un ciclo anual

\begin{tabular}{|c|c|c|c|c|c|c|c|c|}
\hline \multirow{2}{*}{ ÍTEMS PRESA } & \multicolumn{2}{|c|}{ PRIMAVERA } & \multicolumn{2}{|c|}{ VERANO } & \multicolumn{2}{|c|}{ OTOÑO } & \multicolumn{2}{|c|}{ INVIERNO } \\
\hline & $\mathrm{N}$ & $\mathrm{F}$ & $\mathrm{N}$ & $\mathrm{F}$ & $\mathrm{N}$ & $\mathrm{F}$ & $\mathrm{N}$ & $\mathrm{F}$ \\
\hline Rhinella fernandezae & 1 & 4.3 & & & & & 3 & 15.4 \\
\hline Rhinella arenarum & 10 & 4.3 & 4 & 6.2 & & & & \\
\hline \multicolumn{9}{|l|}{ Hylidae } \\
\hline Hypsiboas pulchellus & & & 1 & 6.2 & & & & \\
\hline Hypsiboas sp. & & & 2 & 6.2 & & & & \\
\hline Anura sp. & & & 1 & 6.2 & 1 & 10.0 & 3 & 23.0 \\
\hline \multicolumn{9}{|l|}{ REPTILIA } \\
\hline \multicolumn{9}{|l|}{ Anguidae } \\
\hline Ophiodes vertebralis & 1 & 4.3 & 2 & 12.5 & & & 4 & 23.0 \\
\hline Anguidae sp. & & & & & 1 & 10.0 & & \\
\hline \multicolumn{9}{|l|}{ Colubridae } \\
\hline Liophis poecilogyrus & 1 & 4.3 & & & & & & \\
\hline \multicolumn{9}{|l|}{ AVES } \\
\hline Aves sp. & 2 & 8.7 & 1 & 6.2 & 1 & 10.0 & & \\
\hline
\end{tabular}

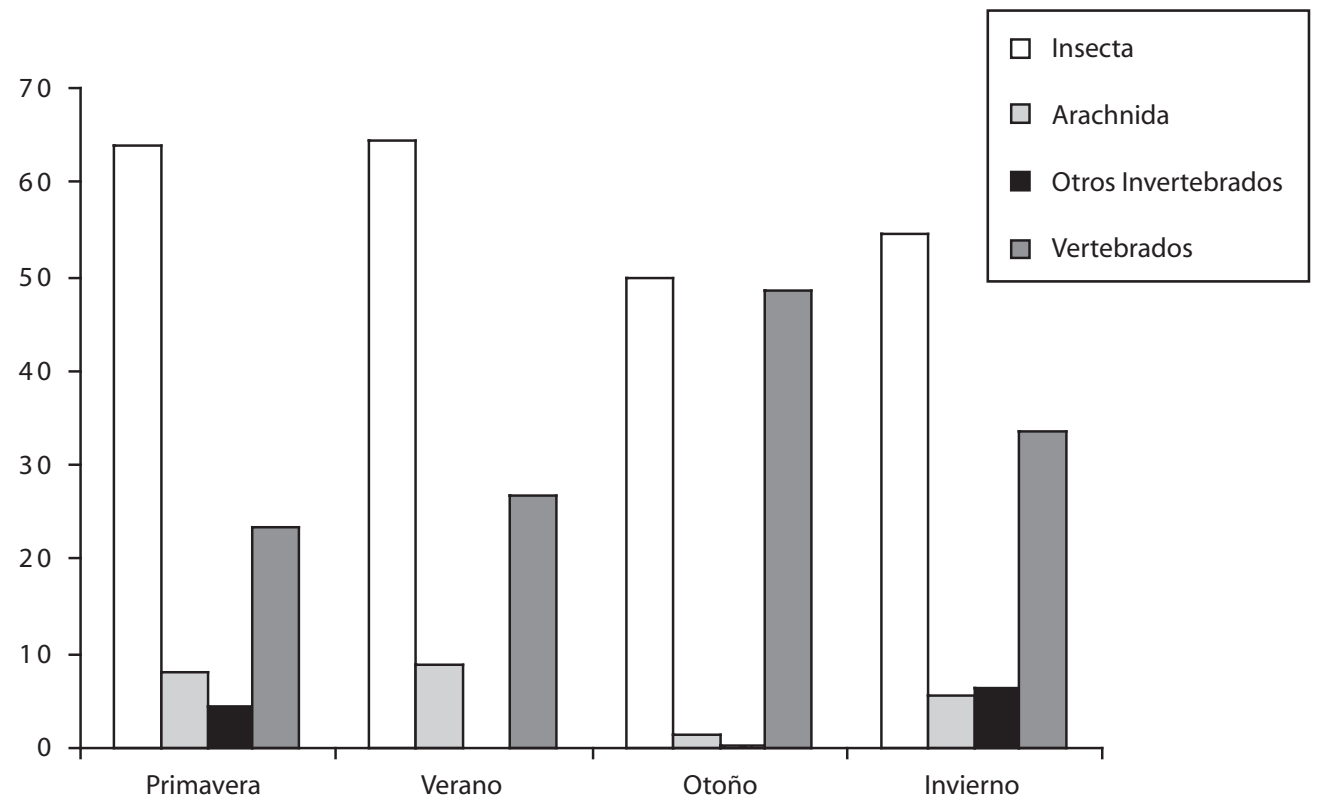

Fig. 2. Contribución en peso (\%) de los ítems presa consumidos durante un año. 
en La Plata (provincia de Buenos Aires), compuesto únicamente por Orthoptera del género Schistocerca. Aravena (1928), sobre la base de siete ejemplares capturados en la provincia de Buenos Aires, determina una dieta $100 \%$ animal, básicamente constituida por larvas de Lepidoptera, Coleoptera, Orthoptera (principalmente tucuras), algunos Hemiptera y un solo Arachnida. Zotta (1934, 1940) sobre la base de doce ejemplares obtenidos principalmente en la provincia de Buenos Aires, registra mayormente Insecta (Hemiptera, Coleoptera, Orthoptera) y también semillas y restos de vertebrados (culebra, roedores, aves).

Recientemente Beltzer (1995) realiza un estudio sobre la alimentación de este Cucúlido en el río Paraná Medio, provincia de Santa Fe (Argentina). Las conclusiones a las que llega este autor son similares a las nuestras en lo que se refiere a la dieta de origen animal, pero la diferencia en el número de entidades taxonómicas registrada en ambos trabajos, es significativa. Beltzer, basándose en la identificación de 1276 presas, registra 39 entidades taxonómicas, mientras que en el presente estudio, con más de 1600 presas, se registraron 93 diferentes ítems. Esto puede deberse, en parte, al escaso muestreo realizado por el mencionado autor, quien obtuvo 22 ejemplares en las cuatro estaciones del año (cuatro en primavera, seis en verano, tres en invierno y nueve en otoño). Este número menor de muestras podría explicar la ausencia de determinados grupos, que en nuestro estudio fueron hallados con frecuencias menores al 1\% (Odonata, Trichoptera, Diptera, Mantodea y Blattodea entre los insectos, Reptiles y Aves entre los Vertebrados). Las diferencias mencionadas y/o la preponderancia de algunos grupos podrían deberse a diferencias geográficas, como es el caso de Hemiptera que representa casi el $80 \%$ de los individuos en el trabajo mencionado, mientras que en nuestra contribución sólo constituyó el $3 \%$.

No se encontraron diferencias significativas entre machos y hembras, tanto en el número como en el peso de las presas consumidas.
En cambio, se registraron variaciones estacionales significativas en el peso de las muestras. En promedio, las más pesadas correspondieron a los meses fríos (otoño y principalmente invierno). Esto puede deberse al consumo de ítems de mayor tamaño (vertebrados), cuando se reduce la disponibilidad de presas menores (insectos y otros invertebrados). En este sentido, la amplitud del nicho trófico es más diversa en los meses cálidos (primavera y verano), coincidiendo con el aumento del número de especies capturadas (desde 29 en invierno hasta 69 en primavera). En la estación invernal el número de especies de algunos taxones (Coleoptera) se redujo significativamente, en tanto otros (larvas de Lepidoptera) aumentaron hasta representar casi el $65 \%$.

El amplio espectro trófico de Guira guira confirma el comportamiento oportunista de esta especie, presentando una selectividad primaria hacia un régimen zoófago.

La importancia del Pirincho como activo destructor de insectos plaga se demuestra por el consumo de especies de interés agrícola y sanitario. Entre los Coleoptera podemos mencionar a Escarabeidae y Elateridae que forman parte del grupo conocido como "gusanos del suelo" y que, en estado larval, causan daño a diversos cultivos. También se registraron Curculionidae fitófagos que atacan productos almacenados. Los Orthoptera más comunes fueron los Acrididae (tucuras), conocidos enemigos de los cultivos tradicionales. Por último, hormigas perjudiciales de los géneros Acromyrmex y Atta (Hymenoptera, Formicidae).

\section{AGRADECIMIENTOS}

A Alda González por la determinación de los Araneida y a Stella Maris Martín por la identificación de los Gastropoda.

\section{RESUMEN}

Se estudió la dieta y ecología alimentaria del ave conocida en Argentina como Pirincho (Guira guira), a 
través del análisis de 62 contenidos estomacales. Las muestras fueron obtenidas entre 1991 y 1997 (23 en primavera, 16 en verano, 10 en otoño y 13 en invierno), en las localidades de La Balandra y Punta Blanca, partido de Magdalena, provincia de Buenos Aires. Ambos sexos fueron analizados en conjunto debido a que no se encontraron diferencias significativas en el número y en el peso de las presas consumidas por machos y hembras (U de Mann-Whitney). La dieta estuvo compuesta en un $100 \%$ por ítemes de origen animal, representada por 92 entidades taxonómicas. Los invertebrados constituyeron más del $96 \%$ de la muestra por número, y los vertebrados un $31 \%$ del peso total. Los insectos fueron hallados en el $98 \%$ de las muestras, representando el $85 \%$ y $59 \%$ en número y peso del total consumido, respectivamente. En los contenidos estomacales se hallaron además anélidos, moluscos, crustáceos, arácnidos, anfibios, reptiles y aves. Estacionalmente no se encontraron diferencias significativas en el número de las presas capturadas. En lo referente al peso, las muestras de invierno resultaron más pesadas que las de primavera y verano (Kruskal-Wallis). El Pirincho es un ave oportunista que consume exclusivamente presas de origen animal.

Palabras clave: Pirincho, Guira guira, alimentación, Buenos Aires, Argentina.

\section{REFERENCIAS}

Aravena, R. O. 1928. Notas sobre la alimentación de las aves. Hornero 4: 153-166.

Beltzer, A. H. 1995. Biología alimentaria del Pirincho Guira guira (Aves: Cuculidae) en el valle aluvial del río Paraná medio, Argentina. Rev. Ecol. Lat. Am. 2: 13-18.

Colwell, R. K. \& D. J. Futuyma. 1971. On the measurements of niche breadth and overlap. Ecology 52: 567-576.

Del Hoyo, J., A. Elliottt \& J. Sargatal. 1997. Handbook of the birds of the world. Volume 4: Sandgrouse to Cuckoos. Lynx, Barcelona, España.

Marelli, C. A. 1919. Sobre el contenido del estómago de algunas aves. Hornero 1: 221-228.

Zotta, A. 1934. Sobre el contenido estomacal de aves argentinas. Hornero 5: 376-383.

Zotta, A. 1940. Lista sobre el contenido estomacal de aves argentinas. Hornero 7: 402-411. 